\title{
ARTICLE
}

\section{Analyses of shielding benchmark experiments using FENDL-3 cross-section data starter library for ITER and IFMIF applications}

\author{
Keitaro Kondo*, Ulrich Fischer, Pavel Pereslavtsev and Arkady Serikov \\ Karlsruhe Institute of Technology (KIT), Eggenstein-Leopoldshafen, 76344, Germany
}

\begin{abstract}
Recently IAEA has been conducting a coordinated research project to update the FENDL-2.1 nuclear data library to FENDL-3. In the present study, FENDL-3, Starter Library, Release 4 (FENDL-3/SLIB4) has been extensively tested through analysis of many fusion relevant benchmark problems. FENDL-3/SLIB4 gives better results for the FNG ITER shielding benchmark and the tungsten benchmark experiments than the previous FENDL-2.1. As for the shielding benchmark in the neutron energy range above $20 \mathrm{MeV}$, the TIARA transmission experiments through iron and concrete using p-Li quasi-monoenergetic neutron source have been analyzed. FENDL-3/SLIB4 provides satisfactory results for the TIARA shielding benchmark through iron. For concrete, however, some discrepancy was found. The present analysis suggests importance of ${ }^{16} \mathrm{O}$ data in the neutron energy range above $20 \mathrm{MeV}$ for shielding calculations through concrete.
\end{abstract}

Keywords: FENDL; nuclear data, fusion neutronics; ITER; IFMIF; shielding; benchmark; Monte Carlo; MCNP

\section{Introduction}

The International Fusion Evaluation Nuclear Data Library (FENDL) is a comprehensive compilation of nuclear cross-section data selected from existing national data libraries to provide a qualified nuclear data base suitable for design analyses of fusion devices. The present version FENDL-2.1, which was up to $20 \mathrm{MeV}$ of incident neutron energy and released in 2004 [1], has been adopted by ITER as the reference library for the nuclear design analyses.

For the past three years the IAEA has been conducting a coordinated research project to develop an extended and updated version of the nuclear data library, FENDL-3 [2]. The present study is devoted to benchmark analyses using the so-called "starter libraries", which have been prepared as a working file to enable benchmark tests for constructing FENDL-3.0. In the FENDL-3.0, many previous evaluations (e.g., ENDF/B-VI, JENDL-3.x) have been replaced by the latest ones. Such analyses are an important step to qualify and validate the new data compilations for a reliable use in important applications for ITER and IFMIF. In this paper we describe the re-analysis of the ITER bulk shield experiment [3] and the tungsten bulk shield experiment [4] previously conducted at the "Frascati Neutron Generator (FNG)" facility of ENEA. The results are compared with calculations using the starter library, release 4 (FENDL-3/SLIB4) and other

*Corresponding author. Email: keitaro.kondo@kit.edu state-of-the-art nuclear data evaluations including JEFF-3.1.1 [5], ENDF/B-VII.0 [6] and JENDL-4.0 [7] as well as the FENDL-2.1 library.

Also one of main objectives of the FENDL-3 project has been to extend the energy range of incident neutrons up to $150 \mathrm{MeV}$ in order to comply with requirements for IFMIF design. Because IFMIF utilizes the d-Li nuclear reaction with deuterons energy of $40 \mathrm{MeV}$, high energy neutrons up to $55 \mathrm{MeV}$ are produced [8]. As for the shielding benchmark in the energy range above 20 $\mathrm{MeV}$, the TIARA transmission experiments through iron and concrete using $\mathrm{p}-\mathrm{Li}$ quasi-monoenergetic neutron source $[9,10]$ have been analyzed using FENDL-3/SLIB4.

\section{Overview of the analyzed benchmark experiments}

\subsection{FNG ITER bulk shield experiment (1995)}

The FNG ITER bulk shield experiment was performed to validate ITER inboard shielding design [4]. The experimental mockup was 94-cm thickness made up of copper, stainless steel/Perspex (polyethylene-type material) sandwich to simulate the first-wall, blanket, vacuum vessel, and toroidal field coils, followed by a smaller block made of alternating plates of copper and stainless steel simulating the magnet. The neutron flux spectra at two positions inside the bulk assembly were measured in the energy range between about $20 \mathrm{keV}$ and $15 \mathrm{MeV}$ with gas-filled proportional counters, stilbene scintillation spectrometer and an NE-213 scintillation 
spectrometer. Also the photon flux spectra were measured with the NE-213 spectrometer for energy range above $0.2 \mathrm{MeV}$.

\subsection{FNG benchmark experiment on tungsten (2001)}

The tungsten benchmark experiment [5] was conducted in order to validate cross sections of tungsten, which is a candidate material for high flux components in the fusion reactor. The dimensions of the $\mathrm{W}$ assembly were $47 \mathrm{~cm} \times 47 \mathrm{~cm} \times 49 \mathrm{~cm}$ and the neutron and photon flux spectra were measured with an NE-213 scintillation spectrometer in four positions (P1-P4).

\subsection{TIARA 40 and $65 \mathrm{MeV}$ neutron transmission benchmark (1996-1997)}

The transmission experiments were performed with collimated 43 and $68 \mathrm{MeV} \mathrm{p-}{ }^{7} \mathrm{Li}$ neutron sources. The test shields of iron up to $130-\mathrm{cm}$ thick and of concrete up to $200-\mathrm{cm}$ thick were located at the end of the collimator and neutron spectra were measured behind the test shield with a BC501A scintillation detector. The source neutron spectra as well as the measured spectra are available in the SINBAD ACCELERATOR database distributed from OECD/NEA.

\section{Calculation method}

The Monte Carlo code MCNP5-1.60 [11] was used for analyses of the experiments. A special source subroutine was used to simulate the source neutron energy and angular distribution of the experiments conducted at FNG. Table 1 shows the original data source of important nuclides contained in stainless steel and concrete as well as tungsten for the FENDL-3/SLIB4 and FENDL-2.1 compilations. The major constituent isotopes in the concrete used in the TIARA transmission benchmark are ${ }^{16} \mathrm{O}(53$ at $\%),{ }^{1} \mathrm{H}$ (19 at\%), ${ }^{28} \mathrm{Si}(13$ at $\%)$ and ${ }^{40} \mathrm{Ca}(5$ at $\%)$.

Table 1. Original data source for FENDL-3/SLIB4 and FE NDL-2.1.

\begin{tabular}{|c|c|c|c|}
\hline \multirow{2}{*}{ Nuclide } & \multicolumn{2}{|c|}{ FENDL-3 } & \multirow{2}{*}{ FENDL-2.1 } \\
\hline & $\leq 20 \mathrm{MeV}$ & $>20 \mathrm{MeV}$ & \\
\hline${ }^{54} \mathrm{Fe}$ & \multicolumn{2}{|c|}{ ENDF/B-VII.1 } & ENDF/B-VI.8 \\
\hline${ }^{56} \mathrm{Fe}$ & JEFF-3.1.1 & TENDL-2011 & JEFF-3 \\
\hline${ }^{57} \mathrm{Fe}$ & \multicolumn{2}{|c|}{ ENDF/B-VII.1 } & ENDF/B-VI.8 \\
\hline${ }^{58} \mathrm{Fe}$ & \multicolumn{2}{|c|}{ JEFF-3.1.1 } & ENDF/B-VI. 8 \\
\hline${ }^{50,52-54} \mathrm{Cr}$ & \multicolumn{2}{|c|}{ KIT-2010 } & ENDF/B-VI.8 \\
\hline${ }^{58,60} \mathrm{Ni}$ & \multicolumn{2}{|c|}{ ENDF/B-VII.1 } & JEFF-3 \\
\hline${ }^{61,62,64} \mathrm{Ni}$ & \multicolumn{2}{|c|}{ ENDF/B-VII.1 } & ENDF/B-VI.8 \\
\hline${ }^{180} \mathrm{~W}$ & \multicolumn{2}{|c|}{ INDL/V-3 } & - \\
\hline${ }^{182-184,186} \mathrm{~W}$ & \multicolumn{2}{|c|}{ INDL/V-3 } & ENDF/B-VI.8 \\
\hline${ }^{1} \mathrm{H}$ & ENDF/B-VII. & JENDL/HE-2007 & JENDL-3.3 \\
\hline${ }^{16} \mathrm{O}$ & \multicolumn{2}{|c|}{ ENDF/B-VII.1 } & ENDF/B-VI.8 \\
\hline${ }^{28} \mathrm{Si}$ & \multirow{2}{*}{\multicolumn{2}{|c|}{ ENDF/B-VII.1 }} & ENDF/B-VI.8 \\
\hline${ }^{40} \mathrm{Ca}$ & & JENDL/HE-2007 & JENDL-3.2 \\
\hline
\end{tabular}

\section{Results and discussions}

\subsection{FNG ITER bulk shield experiment}

The FENDL-3/SLIB4 well reproduces the experimental data of the ITER bulk shield experiment as well as other libraries. Figure 1 shows the comparison of the neutron spectra measured at the position B $(87.6 \mathrm{~cm}$ depth) and calculated with different nuclear data libraries. ENDF/B-VII.0 (cyan) seems to give the slightly smaller flux at the position $\mathrm{B}$, particularly between $0.1-1 \mathrm{MeV}$, although the difference among the libraries is not very large at the position A (41.4 cm depth). Table 2 summarizes the calculation-to-experimental ratios (C/Es) for the neutron flux integrated from 10 to $16 \mathrm{MeV}$, which corresponds to the source neutron energy. The statistical uncertainties in the calculation were less than $0.5 \%$ in all cases. Here one can see there is a difference of $\mathrm{C} / \mathrm{E}$ at the position $\mathrm{B}$ between FENDL-3/SLIB4 and FENDL-2.1; the new compilation gives the better agreement. Table 3 summarizes the $\mathrm{C} / \mathrm{Es}$ for the integrated photon flux. The difference among the calculations with different libraries is not very large, but ENDF/B-VII.0 gives again smaller flux at the position B, probably corresponds to the smaller neutron flux as noticed above. We have confirmed FENDL-3/SLIB4 improves the $\mathrm{C} / \mathrm{E}$ at the position $\mathrm{B}$ as well as JENDL-4.0 compared to previous FENDL-2.1.

Table 2. Calculation-to-Experimental ratios for the neutron flux integrated from 10 to $16 \mathrm{MeV}$ obtained for the ITER bulk shield experiment.

\begin{tabular}{lrc}
\hline Nuclear data & Position A & Position B \\
\hline FENDL-3/SLIB4 & 0.97 & 0.84 \\
FENDL-2.1 & 0.95 & 0.80 \\
JEFF-3.1.1 & 0.97 & 0.84 \\
ENDF/B-VII.0 & 0.96 & 0.79 \\
JENDL-4.0 & 0.99 & 0.86 \\
\hline
\end{tabular}

Table 3. Calculation-to-Experimental ratios for the photon flux integrated from 0.4 to $10 \mathrm{MeV}$ obtained for the ITER bulk shield experiment.

\begin{tabular}{lrc}
\hline Nuclear data & Position A & Position B \\
\hline FENDL-3/SLIB4 & 0.98 & 0.89 \\
FENDL-2.1 & 0.95 & 0.85 \\
JEFF-3.1.1 & 0.93 & 0.85 \\
ENDF/B-VII.0 & 0.93 & 0.82 \\
JENDL-4.0 & 0.99 & 0.91 \\
\hline
\end{tabular}

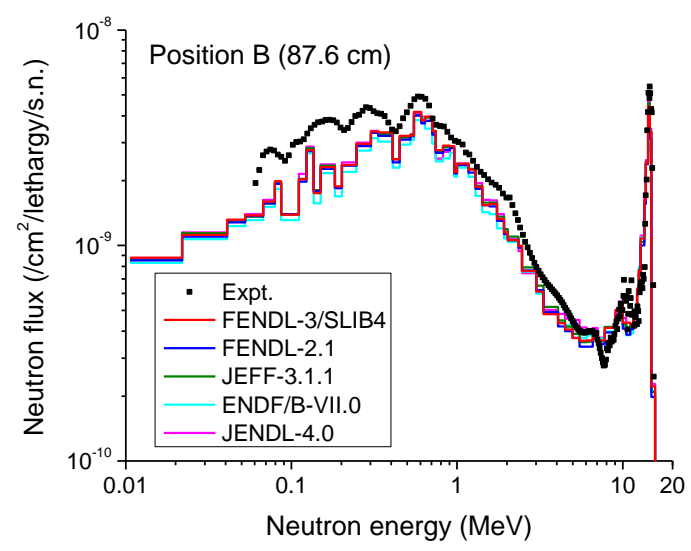

Figure 1. Comparison of the neutron spectra measured at the position B (87.6 cm depth) in the ITER bulk shield experiment and calculated with different nuclear data libraries. 


\subsection{FNG benchmark experiment on tungsten}

Figure 2 shows the comparison of the neutron spectrum measured in the $\mathrm{W}$ benchmark experiment and calculated with different nuclear data libraries. There are two different trends of the calculated spectrum shape between 10 and $14 \mathrm{MeV}$; FENDL-3/SLIB4 and JEFF-3.1.1 reproduce the experimental data well compared to the other libraries. Figure 3 shows the C/Es for the neutron and photon spectra integrated over different ranges of energy. There are large differences among the results with the different libraries in the ranges of 1-5 MeV and 5-10 $\mathrm{MeV}$. In the all ranges FENDL-3/SLIB4 gives better results than FENDL-2.1.

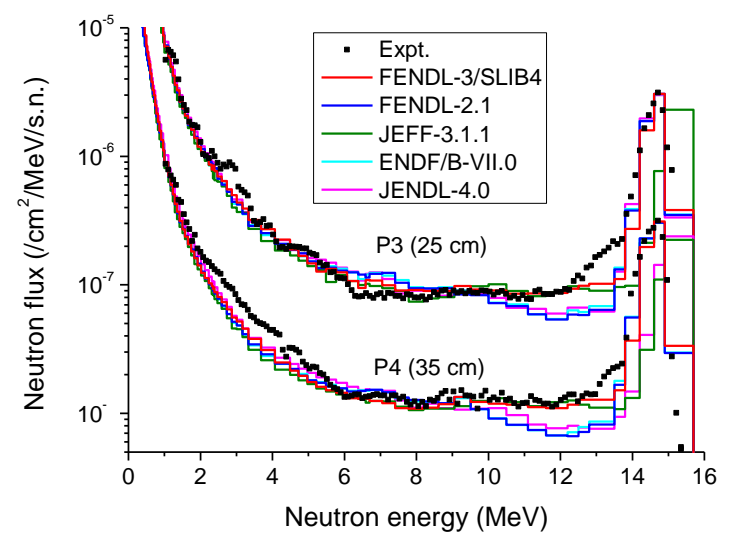

Figure 2. Comparison of the neutron spectra measured in the $\mathrm{W}$ benchmark experiment and calculated with different nuclear data libraries.
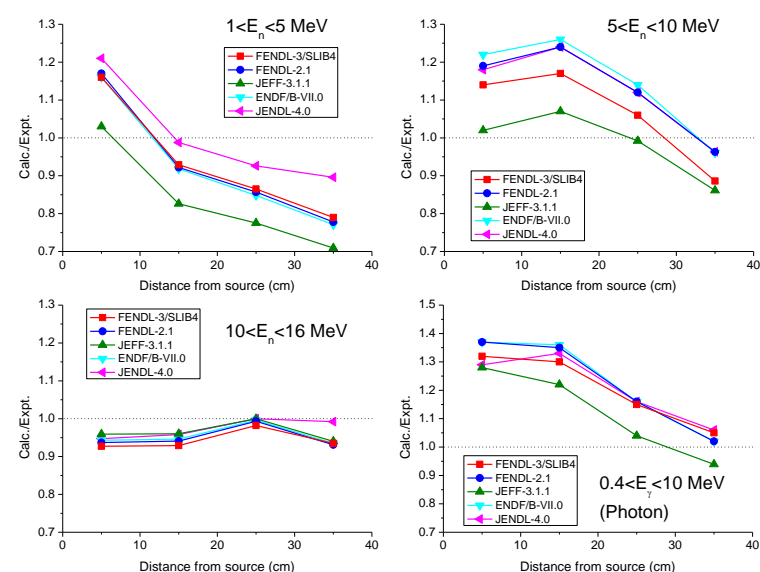

Figure 3. C/E for neutron and photon spectra integrated over different ranges of energy obtained for the $\mathrm{W}$ benchmark experiment.

\subsection{TIARA transmission benchmark experiment}

In the present paper we are focusing on the result for $43-\mathrm{MeV}$ proton incidence due to limitation of space. Figure 4 shows the comparison of the neutron spectra measured and calculated for the TIARA benchmark experiment with the $43 \mathrm{MeV}$ p-Li neutron source through iron, where the libraries used include LA150
[12] and JENDL/HE-2007 ${ }^{\mathrm{a}}$ [13]. As the shield thickness increases, LA150, which was used for previous IFMIF nuclear analyses, significantly overestimates the neutron flux. On the other hand, FENDL-3/SLIB4 reproduces the experimental data very well as well as JENDL/HE-2007.

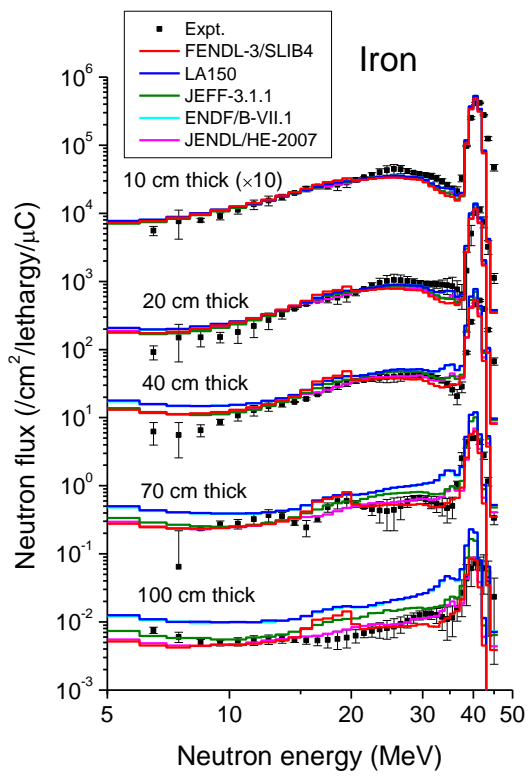

Figure 4. Comparison of the neutron spectra measured and calculated for the TIARA benchmark experiment with 43 $\mathrm{MeV}$ p-Li neutron source through iron.

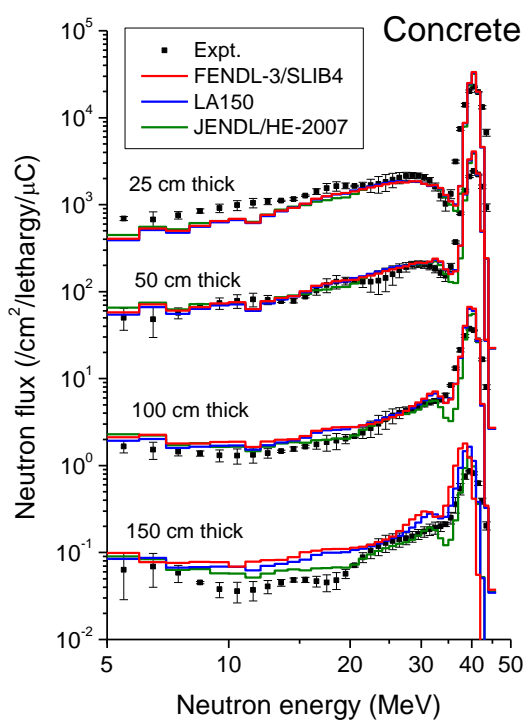

Figure 5. Comparison of the neutron spectra measured and calculated for the TIARA benchmark experiment with 43 $\mathrm{MeV}$ p-Li neutron source through concrete.

Figure 5 shows the comparison of the neutron

${ }^{\text {a }}$ Processed into the ACE format by ourselves with NJOY99.364 with an unofficial patch released from NEA (upnea101_up364). 
spectra measured and calculated for the experiment with the $43 \mathrm{MeV}$ p-Li neutron source through concrete. Only the three libraries are applicable for the analysis because some data above $20 \mathrm{MeV}$ (e.g. ${ }^{1} \mathrm{H}$ in ENDF/B-VII.1, Si in JEFF-3.1.1) are not evaluated in other general purpose libraries. In this comparison FENDL-3/SLIB4 and LA150 show a very similar tendency where the experimental results are overestimated. Although the result with FENDL-3/SLIB4 is on the safe side from the safety point of view, the difference at the peak energy seems fairly large. The difference among calculation may be attributed to the ${ }^{16} \mathrm{O}$ data above $20 \mathrm{MeV}$. Figure 6 shows the comparison of the neutron spectra through concrete of $150 \mathrm{~cm}$ thickness calculated with different ${ }^{16} \mathrm{O}$ nuclear data but FENDL-3/SLIB4 for all other nuclides. The result clearly reveals the large impact of the ${ }^{16} \mathrm{O}$ data on the calculated spectrum. The present analysis suggests the JENDL/HE-2007 evaluation for ${ }^{16} \mathrm{O}$ would be better, though the ENDF/B-VII.1 evaluation is adopted in FENDL-3/SLIB4. Further investigation for the ${ }^{16} \mathrm{O}$ data above $20 \mathrm{MeV}$ is necessary and very important to improve the accuracy of shielding analyses in the intermediate energy range as well as the IFMIF application.

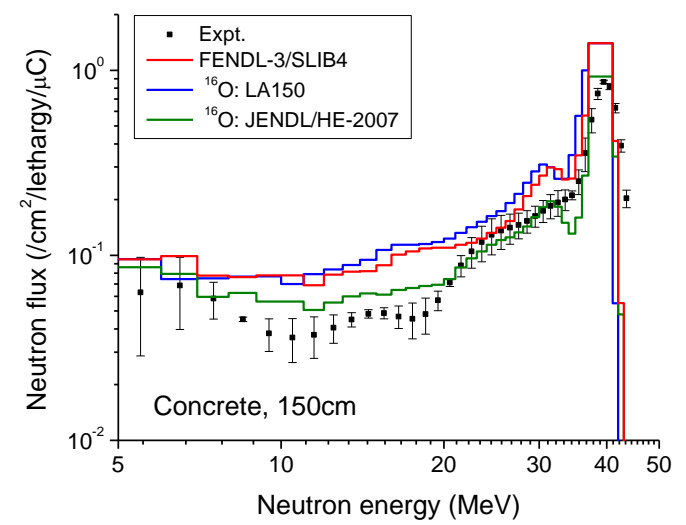

Figure 6. Comparison of the neutron spectra measured and calculated with different ${ }^{16} \mathrm{O}$ nuclear data for the TIARA benchmark experiment with $43 \mathrm{MeV}$ p-Li neutron source through concrete of $150 \mathrm{~cm}$ thickness.

\section{Summary}

FENDL-3, Starter Library, Release 4 (FENDL-3/SLIB4) has been extensively tested by analyzing many fusion relevant benchmark problems. FENDL-3/SLIB4 gives better results for the FNG ITER shielding benchmark and the Tungsten benchmark experiments than previous FENDL-2.1. FENDL-3/SLIB4 provides satisfactory results for the TIARA shielding benchmark through iron. For concrete, however, some discrepancy was found and the calculation overestimated. The present study shows importance of ${ }^{16} \mathrm{O}$ data for the shielding calculation through concrete above the neutron energy of $20 \mathrm{MeV}$ and further investigation focusing on these data is desired.

\section{Acknowledgements}

The framework project of this paper has been funded by the German Ministry for Education and Research (BMBF: Bundesministerium für Bildung und Forschung) under the grant number 03FUS0008, as a contribution to the "Broader Approach" activities. The responsibility for the contents of this publication is with the authors. This work was carried out using the HELIOS supercomputer system at International Fusion Energy Research Centre, Aomori, Japan, under the Broader Approach collaboration between Euratom and Japan, implemented by Fusion for Energy and JAEA.

\section{References}

[1] D. Lopez Al-dama and A. Trokov, FENDL-2.1Update of an evaluated nuclear data library for fusion applications, INDC(NDS)-467, International Atomic Energy Agency, (2004).

[2] http://www-nds.iaea.org/fendl3/

[3] H. Freiesleben, W. Hansen, D. Richter, K. Seidel, $\mathrm{S}$. Unholzer, U. Fischer, Y. Wu, M. Angelone, P. Batistoni and M. Pillon, Neutron and photon flux spectra in a mock-up of the ITER shielding system, Fusion Eng. Des. 47 (1998), pp. 247-253.

[4] K. Seidel, R. Eichin, U. Fischer, R.A. Forrest, H. Freiesleben, D. Leichtle, C. Negoita and S. Unholzer, Benchmark experiments to validate the nuclear data of tungsten, Fusion Eng. Des. 81 (2006), pp. 1211-1217.

[5] A. Santamarina, D. Bernard and Y. Rugama (Ed.), The JEFF-3.1.1 Nuclear Data Library, JEFF Report 22, Nuclear Energy Agency, (2009).

[6] P. Obložinský and M. Herman (Ed.), Evaluated Nuclear Data File ENDF/B-VII.0, Nuclear Data Sheets 107 (2006), pp. 2931-3118.

[7] K. Shibata, O. Iwamoto, T. Nakagawa, N. Iwamoto, A. Ichihara, S. Kunieda, S. Chiba, K. Furutaka, N. Otuka, T. Ohsawa, T. Murata, H. Matsunobu, A. Zukeran, S. Kamada and J. Katakura, JENDL-4.0: A New Library for Nuclear Science and Engineering, J. Nucl. Sci. Technol. 48 (2011), pp. $1-30$.

[8] K. Kondo, F. Arbeiter, U. Fischer, D. Große, V. Heinzel, A. Klix, A. Serikov, K. Tian and V. Weber, Neutronic analysis for the IFMIF target and test cell using a new CAD-based geometry model, Fusion Eng. Des. 87 (2012), pp. 983-988.

[9] N. Nakao, H. Nakashima, T. Nakamura, Sh. Tanaka, Su. Tanaka, K. Shin, M. Baba, Y. Sakamoto and Y. Nakane, Transmission through Shields of Quasi-Monoenergetic Neutrons Generated by $43-$ and $68-\mathrm{MeV}$ Protons. Part-I: Concrete Shielding Experiment and Calculation for Practical Application, Nucl. Sci. Eng. 124 (1996), pp. 228-242.

[10]H. Nakashima, N. Nakao, Sh. Tanaka, T. Nakamura, K. Shin, Su. Tanaka, H. Takada, S. Meigo, Y. Nakane, Y. Sakamoto and M. Baba, Transmission through Shields of 
Quasi-Monoenergetic Neutrons Generated by 43and $68-\mathrm{MeV}$ Protons. Part-II: Iron Shielding Experiment and Analysis for Investigating Calculation Methods and Cross Section Data, Nucl. Sci. Eng. 124 (1996) pp. 243-257.

[11]X-5 Monte Carlo Team, MCNP-A General Monte Carlo N-Particle Transport Code, Version 5, Volume I, MCNP Overview and Theory, LA-UR-03-1987, Los Alamos National Laboratory, (2003).

[12] M.B. Chadwick, P.G. Young, R.E. MacFarlane, P.
Moller, G.M. Hale, R.C. Little, A.J. Koning, and S. Chiba, LA150 Documentation of Cross Sections, Heating, and Damage, LA-UR-99-1222, Los Alamos National Laboratory, (1999).

[13] Y. Watanabe, K. Kosako, S. Kunieda, S. Chiba, R. Fujimoto, H. Harada, M. Kawai, F. Maekawa, T. Murata, H. Nakashima, K. Niita, N. Shigyo, S. Shimakawa, N. Yamano and T. Fukahori, Status of JENDL High Energy File, J. Korean Phys.Soc. 59 (2011), pp. 1040-1045. 